NONCOMMUTATIVE GEOMETRY

AND QUANTUM GROUPS

BANACH CENTER PUBLICATIONS, VOLUME 61

INSTITUTE OF MATHEMATICS

POLISH ACADEMY OF SCIENCES

WARSZAWA 2003

\title{
THE GARDEN OF QUANTUM SPHERES
}

\author{
LUDWIK DĄBROWSKI \\ Scuola Internazionale Superiore di Studi Avanzati \\ Via Beirut 2-4, I-34014 Trieste, Italy \\ E-mail:dabrow@fm.sissa.it
}

Abstract. A list of known quantum spheres of dimension one, two and three is presented.

0. Introduction. Recently, examples of quantum spheres cropped in abundance in the literature. The goal of this note is to aid the book-keeping of these newly emerged species by systematically comparing their basic properties.

As is customary in noncommutative geometry, these quantum spaces are described and studied in terms of certain noncommutative algebras, generalizing the usual correspondence between spaces and function algebras. Here I am concerned mainly with 'deformations' of the *-algebra of polynomials on the sphere $S^{n}$ and their enveloping $C^{*}$-algebras. The $*$-algebras are usually given in terms of generators and relations. Some of these relations can be regarded as deformations of the commutation relations and some as deformations of the sphere relation $\sum_{j=1}^{n+1} x_{j}^{2}=1$. The classical spheres are often particular members of the family, or 'limit' cases. Here, by the dimension of such a quantum sphere I understand just the number $n$.

In this note the examples in lowest dimensions (one, two and three) are listed (in alphabetical order). They appeared in the literature known to me, and I do not guarantee that the list is complete. Most of them have a $C^{*}$-algebraic version and often the deformation forms a continuous field of $C^{*}$-algebras. The smooth structure is described only in a few cases but basic properties such as classical subspaces (sets of characters) and $C^{*}$-algebraic $\mathrm{K}$-groups are given most of the time.

It turns out that among basic building blocks of quantum spheres are noncommutative tori and discs. The $C^{*}$-algebra of the noncommutative torus $T_{\theta}^{2}, 0 \leq \theta<1$, is generated

2000 Mathematics Subject Classification: 81R60, 81R50, 20G42, 58B34, 58B32, $17 \mathrm{~B} 37$.

Key words and phrases: noncommutative geometry, quantum spheres.

A financial support of 'Geometric Analysis' Research Training Network HPRN-CT-199900118 of E. C. is kindly acknowledged.

The paper is in final form and no version of it will be published elsewhere. 
by two unitaries $U, V$ with the relation $U V=e^{2 \pi i \theta} V U$, see [49] for an extensive study. It carries a natural action of the usual torus $T^{2}=S^{1} \times S^{1}$ and thus also of $\mathbb{R}^{2}$. The $C^{*}$-algebra of a quantum disc $D_{\mu, q}, 0 \leq \mu \leq 1,0<q \leq 1$, is generated by $z$ with the relation $q z z^{*}-z^{*} z=q-1+\mu\left(1-z z^{*}\right)\left(1-z^{*} z\right) \quad$ [31]. For $0 \leq \mu<1-q$ and for $q=1,0<\mu<1$, they are known [31, 52] (see also [25] and references therein) to be all isomorphic to the Toeplitz algebra $\mathcal{T}$ and, in turn, also to the $C^{*}$-algebra generated by the raising (shift) operator $e_{j} \mapsto e_{j+1}$ on $\ell^{2}(\mathbb{N}),[52]$.

All the algebras of quantum spheres considered in the sequel are unital and the algebra unit is denoted as $I$. $\mathcal{K}$ stands for the compact operators on an infinite-dimensional separable Hilbert space.

\section{Quantum circle}

\subsection{Markov [34].}

Generators: $A, B$. Relations:

$$
A=A^{*}, \quad B=B^{*}, \quad A^{2}+B^{2}=I .
$$

Classical subset: $S^{1}$. K-groups: $K_{0}=\mathbb{Z}, K_{1}=0$ [40].

\section{Remarks:}

1. The universal $C^{*}$-algebra for these relations can be represented as the algebra of all continuous $2 \times 2$ matrix-valued functions on the rectangle $[0, \pi / 2] \times[0, \pi]$ satisfying certain boundary conditions.

2. The isomorphic universal unital $C^{*}$-algebra of the quantum disc at $q=-1, \mu=0$ (generated by $z$ with the relation $z z^{*}+z^{*} z=2 I$ ) has been independently studied in [41].

3. Note that this example does not really fit to our list because there is no deformation parameter. However, it could be supplemented by a commutation relation, e.g., $A B=q B A$, with $q= \pm 1$. Then the case $q=1$ corresponds to the classical $S^{1}$ while in the case $q=-1$ the $C^{*}$-algebra is isomorphic to the universal $C^{*}$-algebra of the free product of groups $Z_{2} * Z_{2}$ with $K_{0}=\mathbb{Z}^{3}, K_{1}=0$ and the classical subspace consisting of four points.

\section{Quantum 2-spheres}

\subsection{Bratteli, Elliott, Evans, Kishimoto [3, 4, 5].}

Generators: $A, B$. Parameter: $-1<\lambda=\cos (2 \pi \theta)<1, \quad(0<\theta<1 / 2)$. Relations:

$$
\begin{aligned}
A=A^{*}, & B=B^{*}, \\
B A B A= & \left(4 \lambda^{2}-1\right) A B A B-2 \lambda A^{2} B^{2}+8 \lambda\left(1-\lambda^{2}\right)\left(A^{2}+B^{2}-I\right), \\
A^{2} B+B A^{2}= & 2 \lambda A B A+4\left(1-\lambda^{2}\right) B, \\
A B^{2}+B^{2} A= & 2 \lambda B A B+4\left(1-\lambda^{2}\right) A .
\end{aligned}
$$

Classical subset: $\emptyset . \quad \underline{\text { K-groups: }} K_{0}=\mathbb{Z}^{6}, K_{1}=0[45,32]$. 


\section{Remarks:}

1. Introduced, via $A=U+U^{-1}, B=V+V^{-1}$, as a fixed point algebra of the 'flip' automorphism $\sigma: U \mapsto U^{-1}, V \mapsto V^{-1}$ of the noncommutative torus $T_{\theta}^{2}$.

2. Classically (for $\theta=0$ ) this is a 'pillow' (a smooth 2-sphere with four corners coming form the fixed points of $\sigma$ ). After the deformation (for $0<\theta<1 / 2$ ) this geometry manifests itself in $K_{0}=\mathbb{Z}^{6}$ (with four generators besides $I$ and the Bott projector).

3. If $\lambda=1(\theta=0)$ the (unital) universal $C^{*}$-algebra for the relations above is indeed isomorphic to $C\left(S^{2}\right)$ because it turns out that in this case either of the last two relations implies that $A B=B A$ (see [3], p. 150). Then the middle relation is spurious and $S^{2}$ arises as a one point compactification of $\mathbb{R}^{2}$.

4. For irrational $\theta$ these $C^{*}$-algebras are simple, approximately finite-dimensional and with a unique trace state.

5. A closely related (strongly Morita equivalent for $\theta \neq 1 / 2$ ) is the crossproduct $C^{*}$-algebra $T_{\theta}^{2} \rtimes_{\sigma} \mathbb{Z}_{2}$, where the generator of $\mathbb{Z}_{2}$ acts on the noncommutative torus $T_{\theta}^{2}$ by the 'flip' automorphism $\sigma: U \mapsto U^{-1}, V \mapsto V^{-1}$, cf. [3, 4, 5, 32, 57]. It is generated by three unitaries $U, V, W$ with the relations $V U=e^{2 \pi i \theta} U V, W U W=$ $U^{*}, W V W=V^{*}, W^{2}=I$. For irrational $\theta, \theta^{\prime}$ these $C^{*}$-algebras are isomorphic iff $\theta=\theta^{\prime}$ or $\theta=1-\theta^{\prime}$. For rational $\theta=p / q, \theta^{\prime}=p^{\prime} / q^{\prime}$, with $p, p^{\prime}$ and also $q, q^{\prime}$ relatively prime, they are isomorphic iff $q=q^{\prime}$.

6. This algebra admits other presentations [32] with three selfadjoint unitaries $X, Y, Z$ and the relation $X Y Z=e^{2 \pi i \theta} Z Y X$, or with four selfadjoint unitaries $X, Y, Z, T$ and the relation $X Y=e^{\pi i \theta} T Z$.

2.2. Calow, Matthes [11].

Generators: $A, B$. Parameters: $0<p, q<1$. Relations:

$$
\begin{aligned}
A & =A^{*}, \\
B^{*} B-q B B^{*} & =(p-q) A+(1-p) I, \\
A B-p B A & =(1-p) B \\
(I-A)\left(B B^{*}-A\right) & =0 .
\end{aligned}
$$

Classical subset: $S^{1}$. K-groups: $K_{0}=\mathbb{Z}^{2}, K_{1}=0[11]$.

Remarks:

1. Obtained (at the *-algebra level) by glueing the quantum disc $D_{0, p}$ with $D_{0, q}$.

2. Classically (for $p=1=q$ ) this is a commutative $*$-algebra, which with the additional conditions $\|A\|=1$ and $\|B\|=1$, describes a closed cone with one vertex and one circular edge (topologically $S^{2}$ ). The last two conditions are automatic if $p \neq 1, q \neq 1$.

3. It is not $*$-isomorphic [26], p. 6 , Th. 1.4, with any of the Podleś quantum spheres 2.5. However, its universal $C^{*}$-algebra is isomorphic to the $C^{*}$-algebra of the generic $(s>0)$ Podleś quantum sphere 2.5.5.

\subsection{Gurevich, Leclercq, Saponov [24].}

Generators: $A, B . \underline{\text { Parameters: }} h \geq 0, q>0$. Relations: 


$$
\begin{gathered}
A=A^{*}, \quad q B^{*} B+q^{-1} B B^{*}+q^{2}\left(q+q^{-1}\right) A^{2}=\left(q+q^{-1}\right) I, \\
q^{2} A B-B A=h B, \quad B B^{*}-B^{*} B=\left(1-q^{4}\right) A^{2}+h\left(1+q^{2}\right) A .
\end{gathered}
$$

Classical subset: $S^{2}$ for $h=0, q=1 ; S^{1}$ for $h=0, q \neq 1$; a point for $\pm\left(q^{4}-1\right) / q=$ $h \neq 0$ and $\emptyset$ otherwise.

Remarks:

1. I have set the (radius) parameter $-\alpha=1$ in [24] by overall rescaling of the generators, which relate to those used in [24] as $B=b$ and $A=g /\left(1+q^{2}\right)$, and the parameters as $h=q^{-1} \hbar$ ( $q$ is unchanged).

2. This example deforms the universal enveloping algebra of $s u(2)$ with a constrained value of the quadratic Casimir element.

3. The $C^{*}$-algebra completion is in general not possible but can be accomplished in particular cases.

4. The one-dimensional subfamily with $h=0$ coincides with the one parameter subfamily of equatorial quantum Podleś [48] spheres 2.5.6.

5. Another particular one-dimensional subfamily with $q=1$ coincides, using the variables $Z=B / \sqrt{h^{2}+4}, H=A / \sqrt{h^{2}+4}$ and $\mu=h / \sqrt{h^{2}+4}$ (when treated as a formal parameter) with the quantum 2-sphere of Omori, Maeda, Miyazaki, Yoshioka [46]. Any member of this subfamily forms a basis of a quantum principal $U(1)$-bundle in the sense of Hopf-Galois theory [7] with a total space being a formal deformation of $S^{3}$ by a non-central formal parameter $\mu$, cf. Section 4 .

\subsection{Natsume, Olsen [42], [44].}

Generators: $A, B . \quad$ Parameter: $t \in \mathbb{R} . \quad \underline{\text { Relations: }}$

$$
A=A^{*}, B^{*} B+A^{2}=I, B B^{*}+\left(t B B^{*}+A\right)^{2}=I, B A-A B=t B B^{*} B .
$$

Classical subset: two points for $t \neq 0$. K-groups: $K_{0}=\mathbb{Z}^{2}, K_{1}=0$ [44].

\section{Remarks:}

1. Motivated by Poisson geometry.

2. For $t \in\left[0,1 / 2\right.$ [ the enveloping $C^{*}$-algebras are of type I and are isomorphic [44] to certain extension of $\mathbb{C}^{2}$ by the crossproduct $C^{*}$-algebra $C_{0}(]-1,1[) \rtimes_{\alpha_{t}} \mathbb{Z}$, or equivalently by $\mathcal{K} \otimes C\left(S^{1}\right)$. Here the generator of $\mathbb{Z}$ acts by an automorphism $\alpha_{t}$ given by the pull-back of the homeomorphism $\left.x \mapsto t x^{2}+x-t, \forall x \in\right]-1,1[$, which is topologically conjugate to the translation by 1 on $\mathbb{R}[44]$.

3. They form a continuous field of $C^{*}$-algebras over $[0,1 / 2[$, which is trivial over ] $0,1 / 2[$. In particular they are all isomorphic for $t \in] 0,1 / 2[$. They also constitute a strong deformation of $C\left(S^{2}\right)[44]$.

\subsection{Podleś [48].}

Generators: $A, B . \quad$ Parameters: $0 \leq q<1,0 \leq s \leq 1$.

Relations:

$$
\begin{gathered}
A^{*}=A, \quad B A=q^{2} A B \\
B B^{*}=-q^{4} A^{2}+\left(1-s^{2}\right) q^{2} A+s^{2} I, \quad B^{*} B=-A^{2}+\left(1-s^{2}\right) A+s^{2} I .
\end{gathered}
$$


Classical subset: a point if $s=0 ; S^{1}$ if $\left.\left.s \in\right] 0,1\right]$. K-groups: $K_{0}=\mathbb{Z}^{2}, K_{1}=0$ [36]. Remarks:

1. Discovered as homogeneous $S U_{q}(2)$-spaces.

2. In order to write the relations of the whole family in a uniform way, a parameter $0 \leq s \leq 1$ is used here. It relates to the parameter $0 \leq c \leq \infty$ in [48] by $s=$ $2 \sqrt{c} /\left(1+\sqrt{1+4 c}\right.$ ) (and $\left.c=\left(s^{-1}-s\right)^{-2}\right)$. Also, the Podleś generators $A, B$ are rescaled, iff $0 \leq s<1$ (i.e., $0 \leq c<\infty$ ), by $1-s^{2}$.

3. Any member of this family describes a 'round' quantum sphere, in the sense that there exist Cartesian coordinates, i.e. three selfadjoint elements generating the *-algebra such that their squares sum up to $I$ and all other relations are only commutation relations. In particular they commute for $q=1$.

4. The case $s=0$ (i.e., $c=0$ ) when the last two relations read $B B^{*}=q^{2} A-q^{4} A^{2}$ and $B^{*} B=A-A^{2}$, is known as the standard Podles sphere. It can be viewed as a quotient sphere $S U_{q}(2) / U(1)$ in the spirit of the Hopf fibration (cf. Example 3.4). For all $0 \leq q<1$, the corresponding $C^{*}$-algebras are isomorphic to the minimal unitization of the compacts $\mathcal{K}$. This $C^{*}$-algebra can be obtained by deformation quantization of Rieffel [50], by noting that $S^{2}$ with one point removed admits an action of $\mathbb{R}^{2}$.

5. For $0<s \leq 1$ (i.e., $0<c \leq \infty$ ) the related $C^{*}$-algebras are all isomorphic [52] to an extension of $C\left(S^{1}\right)$ by $\mathcal{K} \oplus \mathcal{K}$, or an extension of the Toeplitz algebra $\mathcal{T}$ by $\mathcal{K}$. This $C^{*}$-algebra can be obtained as the deformation quantization of Rieffel [50] by noting that $S^{2}$ with a circle removed admits an action of $\mathbb{R}^{2}$. It can also be viewed as the $C^{*}$-algebra of two quantum discs glued along their boundaries $S^{1}$ ([52], cf. $[10,11])$, as the Cuntz-Krieger algebra of a certain graph [30], or as the quantum double suspension of two points [30].

6. The case $s=1$ (i.e., $c=\infty$ ) when the last two relations read $B B^{*}=-q^{4} A^{2}+I$ and $B^{*} B=-A^{2}+I$, is known as the equatorial Podleś sphere. It is easily seen to be *-isomorphic to the two-dimensional Euclidean sphere introduced in [22]. As such, it admits a higher (even) dimensional generalization. Also, it contains the $*$-algebra of quantum disk, which can be geometrically interpreted as collapsing this quantum 2 -sphere by the reflection with respect to the equatorial plane [25]. Moreover, it is isomorphic to the quotient of the underlying *-algebra of Example 3.4. (for the parameter $q^{2}$ ) by the relation $b=b^{*}$. The geometric meaning of this is that the equatorial Podleś sphere embeds as an equator in $S U_{q^{2}}(2)$ thought of as a quantum 3 -sphere [27]. Hence for fixed $q$, the path $0 \leq s \leq 1$ of Podleś spheres can be viewed as an interpolation between the quotient sphere $S U_{q}(2) / U(1)$ and the embedded (equator) 2-sphere in $S U_{q^{2}}(2)$.

\section{Quantum 3-spheres}

\subsection{Calow, Matthes [12].}

Generators: $a, b . \quad$ Parameters: $0<p, q<1$. Relations:

$$
a^{*} a-q a a^{*}=(1-q) I, b^{*} b-p b b^{*}=(1-p) I, a b=b a, a^{*} b=b a^{*},\left(I-a a^{*}\right)\left(I-b b^{*}\right)=0 .
$$


Classical subset: $S^{1} \times S^{1} . \quad$ K-groups: $K_{0}=\mathbb{Z}, \quad K_{1}=\mathbb{Z}[26]$. Remarks:

1. As a $*$-algebra it is obtained by glueing the quantum solid tori $D_{0, p} \times S^{1}$ and $D_{0, q} \times S^{1}$.

2. As a $C^{*}$-algebra it is isomorphic to $(\mathcal{T} \otimes \mathcal{T}) /(\mathcal{K} \otimes \mathcal{K})$. The latter is the Cuntz-Krieger algebra of a certain graph of rank two [26].

3. Classically (for $p=1=q$ ) this $C^{*}$-algebra with additional conditions $\|a\|=1$ and $\|b\|=1$ (which are automatic if $p \neq 1, q \neq 1$ ), is isomorphic to $C\left(S^{3}\right)$.

4. It forms a locally trivial, globally nontrivial [12], in fact noncleft [26], quantum principal $U(1)$-bundle (Hopf-Galois extension) over the quantum $S^{2}$ of Example 2.2.

3.2. Connes, Dubois-Violette [16].

Generators: $x^{0}, x^{1}, x^{2}, x^{3}$.

$$
\begin{aligned}
x^{0}=\left(x^{0}\right)^{*}, \quad x^{1}=\left(x^{1}\right)^{*}, & x^{2}=\left(x^{2}\right)^{*}, \quad x^{3}=\left(x^{3}\right)^{*}, \\
\cos \left(\varphi_{1}\right)\left(x^{0} x^{1}-x^{1} x^{0}\right) & =i \sin \left(\varphi_{2}-\varphi_{3}\right)\left(x^{2} x^{3}+x^{3} x^{2}\right), \\
\cos \left(\varphi_{2}\right)\left(x^{0} x^{2}-x^{2} x^{0}\right) & =i \sin \left(\varphi_{3}-\varphi_{1}\right)\left(x^{3} x^{1}+x^{1} x^{3}\right), \\
\cos \left(\varphi_{3}\right)\left(x^{0} x^{3}-x^{3} x^{0}\right) & =i \sin \left(\varphi_{1}-\varphi_{2}\right)\left(x^{1} x^{2}+x^{2} x^{1}\right), \\
\cos \left(\varphi_{2}-\varphi_{3}\right)\left(x^{2} x^{3}-x^{3} x^{2}\right) & =-i \sin \left(\varphi_{1}\right)\left(x^{0} x^{1}+x^{1} x^{0}\right), \\
\cos \left(\varphi_{3}-\varphi_{1}\right)\left(x^{3} x^{1}-x^{1} x^{3}\right) & =-i \sin \left(\varphi_{2}\right)\left(x^{0} x^{2}+x^{2} x^{0}\right), \\
\cos \left(\varphi_{1}-\varphi_{2}\right)\left(x^{1} x^{2}-x^{2} x^{1}\right) & =-i \sin \left(\varphi_{3}\right)\left(x^{0} x^{3}+x^{3} x^{0}\right), \\
\left(x^{0}\right)^{2}+\left(x^{1}\right)^{2}+\left(x^{2}\right)^{2}+\left(x^{3}\right)^{2} & =I .
\end{aligned}
$$

Classical subset: generically discrete. K-groups: will be studied in part II of [16]. Remarks:

1. This family of $*$-algebras has been obtained by requiring that the $2 \times 2$ matrix

$$
u=\left(\begin{array}{cc}
x^{0}+i e^{i \varphi_{3}} x^{3} & i e^{i \varphi_{1}} x^{1}+e^{i \varphi_{2}} x^{2} \\
i e^{i \varphi_{1}} x^{1}-e^{i \varphi_{2}} x^{2} & x^{0}-i e^{i \varphi_{3}} x^{3}
\end{array}\right)
$$

is unitary and its entries $u_{j k}$ satisfy $c h_{1 / 2}(u):=\sum_{j, k=1}^{2}\left(u_{j k} \otimes u_{k j}^{*}-u_{j k}^{*} \otimes u_{k j}\right)=0$.

2. The particular one parameter subfamily, $\varphi_{1}=\varphi_{2}=-\frac{1}{2} \theta$ and $\varphi_{3}=0$, appeared in [17]. It coincides, using the variables $Z=x^{0}+i x^{3}, W=x^{1}+i x^{2}$, with the particular one parameter subfamily of Matsumoto quantum spheres 3.3 , when $\Theta=\theta$ is a constant function and also with the Natsume, Olsen 3-spheres [43]. The related $C^{*}$-algebras can be obtained as $\theta$-deformation by noting that $S^{3}$ admits an action of the ordinary torus, cf. Section 4 , and thus as a particular case of the deformation quantization of Rieffel [50] (cf. [56, 54]). Any member of this subfamily fulfills all the properties of a noncommutative manifold in the sense of [14] and has a higher (odd) dimensional generalization [43].

3.3. Matsumoto [37, 38].

Generators: a pair $Z, W$ of normal operators on a Hilbert space.

Parameters: real valued continuous functions $\Theta$ on the closed interval $[0,1]$. 
Relations:

$$
Z W=e^{2 \pi i \widehat{\Theta}\left(Z^{*} Z\right)} W Z, \quad Z^{*} Z+W^{*} W=I,
$$

where $\widehat{\Theta}\left(Z^{*} Z\right)$ stands for the self-adjoint operator obtained by the functional calculus from the operator $Z^{*} Z$ and a continuous function $\Theta$.

Classical subset: $\left(\left(\Theta^{-1}(\mathbb{Z}) \cap\right] 0,1[) \times T^{2}\right) \cup\left(\left(\Theta^{-1}(\mathbb{Z}) \cap\{0,1\}\right) \times S^{1}\right)$.

K-groups: $K_{0}=\mathbb{Z}, K_{1}=\mathbb{Z}$.

Remarks:

1. Obtained by glueing two quantum solid tori described by the crossproduct $C^{*}$ algebra $\mathcal{C}(D) \rtimes_{\Theta} \mathbb{Z}$, where the generator of $Z$ acts on the 2-disk $D$ as a rotation by angle $\Theta(r)$, and $r \in[0,1]$ is the coordinate on $D$.

2. In the sense of [21, Definition 2.4], forms a quantum principal $U(1)$-bundle (Hopf fibration) over the usual 2-sphere. This can be easily verified much in the same way as in [26, Remark ].

3. When the function $\Theta$ is a constant number $\theta$, the $C^{*}$-algebra generated by the relations above is isomorphic to the universal $C^{*}$-algebra generated by two normal operators $T, S$ satisfying $T S=e^{2 \pi i \theta} S T,\left(I-T^{*} T\right)\left(I-S^{*} S\right)=0$ and $\|T\|=1=$ $\|S\|$, introduced in [37]. Its classical subset is $S^{1} \sqcup S^{1}$ and it has odd-dimensional generalization due to Natsume and Olsen [43]. It coincides with the particular one parameter subfamily $\varphi_{1}=\varphi_{2}=-\frac{1}{2} \theta$ and $\varphi_{3}=0$ of Connes, Dubois-Violette [16].

\subsection{Woronowicz [58].}

Generators: $a, b . \quad$ Parameters: $q \in \mathbb{C}$. Relations:

$$
b a=q a b, a^{*} b=q b a^{*}, a a^{*}+b b^{*}=I, a^{*} a+|q|^{2} b b^{*}=I, b b^{*}=b^{*} b .
$$

Classical subset: $S^{1}$ for $|q| \neq 1, S^{1} \sqcup S^{1}$ for $|q|=1$ and $q \neq 1, S^{3}$ for $q=1$.

K-groups: $K_{0}=\mathbb{Z}, K_{1}=\mathbb{Z}$ for $q>0[35]$.

Remarks:

1. Discovered as a family of compact matrix quantum groups $S U_{q}(2)$ for $-1 \leq q \leq 1$, $q \neq 0$.

2. Here I allow the range of the parameter to $q$ to be complex. This 'interpolates' between the original Woronowicz family $q \in \mathbb{R}$, and the one-parameter subfamily $|q|=$ 1 which coincides (if $q=e^{i \theta}$ ) with Natsume and Olsen [43] family and also with the particular one parameter subfamily $\varphi_{1}=\varphi_{2}=-\frac{1}{2} \theta$ and $\varphi_{3}=0$ of Example 3.2 of Connes, Dubois-Violette [16]. The transformation $a \mapsto a^{*}, b \mapsto-q b$, and $q \mapsto 1 / q$ defines a $*$-isomorphism for $q \neq 0$, hence it suffices to restrict to the range $|q| \leq 1$.

3. For $0 \leq q<1$ the members of this family are easily seen to be $*$-isomorphic to the three dimensional Euclidean spheres introduced in [22] and also to the three dimensional unitary spheres introduced as quantum homogeneous spaces of $S U_{q}(n)$ in [55]. It turns out that also their higher dimensional generalizations, the quantum Euclidean spheres and the quantum unitary spheres, are $*$-isomorphic at any given odd dimension. ${ }^{1}$

${ }^{1}$ To our knowledge, this simple fact, which was observed during a conversation with F. Bonechi, E. Hawkins and G. Landi, has not been presented before. 
4. For all $q \in \mathbb{C}$ these $*$-algebras have a $C^{*}$-algebraic version. For $q=1$ this is just $C\left(S^{3}\right)$. The case $q=-1$ has been studied in [59]. For $0 \leq q<1$ these $C^{*}$-algebras are all isomorphic to a certain extension of $C\left(S^{1}\right)$ by $C\left(S^{1}\right) \otimes \mathcal{K}$, which can also be described as the Cuntz-Krieger algebra of a certain graph [30], or as the quantum double suspension of the circle [30].

5. For $0<q \leq 1, S U_{q}(2)$ forms a quantum principal $U(1)$-bundle (Hopf fibration) over the Podleś quantum 2-sphere of Example 2.5 in the sense of Hopf-Galois extensions (if $s=0$ ) or coalgebra-Galois extensions (if $s \in] 0,1]$ ), see [6], cf. [9].

6. For some further noncommutative-geometric aspects see [15] and references therein.

4. Final comments. Some finite dimensional algebras (in a sense corresponding to zero dimensional quantum spaces but, nevertheless, possessing certain properties of 2-spheres) have also been studied. For instance, the classification [48] of $S U_{q}(2)$-homogeneous spaces, besides the family 2.5 of Podleś quantum spheres, also includes a discrete series of full matrix algebras Mat $_{N}$. It has been observed in [23] that this family of 'quantum spheres' can be equipped with an additional structure, notably a sequence of injections Mat $_{N} \rightarrow$ Mat $_{N+1}$, which are morphisms in the category of $U_{q}(s u(2))$-modules. For $q=1$, this agrees with the fuzzy-sphere philosophy of [33]. Therein, the $N \times N$ matrix algebras are considered as $U(s u(2))$-modules together with $U(s u(2))$-module injections that form a direct system whose limit is the algebra of polynomials on $S^{2}$ [29]. One can also show that the matrix algebras converge to the sphere for the quantum Gromov-Hausdorff distance [51]. Furthermore, these matrix algebras can be viewed as representations of the universal enveloping algebra of $s l(2)$ with the value $\frac{N^{2}-1}{4}$ of the quadratic Casimir element. The discrete family of Podleś spheres can be thought of as the family of q-fuzzy spheres [23]. (The aforementioned injections are $U_{q}(s u(2))$-linear.)

There are other examples of quantum spheres which do not fit exactly our list as they are not deformations in our sense (families of $*$-algebras). In [46, 47] a formal deformation of $S^{3}$ (as a contact manifold) is provided with a invertible non-central deformation 'parameter' $\mu$, generators $a, b$ and relations

$$
\begin{gathered}
\mu=\mu^{*}, a^{*} a+b^{*} b=I, \mu^{-1} a-a \mu^{-1}=-a, \mu^{-1} b-b \mu^{-1}=-b, \\
b a=a b, a b^{*}=(1-\mu) b^{*} a, a a^{*}-(1-\mu) a^{*} a=\mu, b b^{*}-(1-\mu) b^{*} b=\mu .
\end{gathered}
$$

This deformation yields a certain 'smooth' algebra admitting a $U(1)$-action that is principal in the sense of Hopf-Galois theory [7]. (The base space of this quantum pricipal bundle is given by the quantum 2-sphere of Example 2.3.5.) Another example of a noncommutative Hopf fibration given by a principal $U(1)$-action on a super 3 -sphere was studied in [18]. There are also examples of quantum complex spheres related to the Jordanian quantum group $S L_{h}(2)$ [13, 60].

As far as four-dimensional quantum spheres are concerned, recently several examples, together with instanton bundles over them, have been constructed. They indicate a wealth even greater than that of the known one, two and three dimensional examples.

It should be mentioned that various principles were employed to proliferate the examples of quantum spheres. They were coming, e.g., from the Poisson, contact or homogeneous structure. We have also encountered several types of glueing and quotienting. 
One of the tools is the usual suspension operation which can be used to find links between different examples and to produce new examples of one dimension greater. Note, for instance, that the suspension of Example 3.2.2 is just the Connes and Landi noncommutative 4-sphere [17], while the suspension of Example 3.4 occurs in [19], (see also $[20])$. A $C^{*}$-algebraic noncommutative double suspension has been mentioned in examples 2.2, 2.5 and 3.4. A kind of quantum double suspension at the $*$-algebra level, which raises the dimension by two and yields different families of quantum 4-spheres, has been employed in [53] and [8]. The example of a quantum 4-sphere presented in [1], with its classical subspace being just a point, is yet another type of a double suspension [2] of the standard Podleś quantum sphere. It is motivated by the Poisson structure. Another method to obtain more examples is a $\theta$-deformation of a manifold $M$ carrying an action of the usual torus $T^{2}$. The related $C^{*}$-algebra is constructed as the fixed-point algebra for the diagonal action of $T^{2}$ on the tensor product of $C(M)$ with the algebra of the noncommutative torus $T_{\theta}^{2}$. This is a particular instance of a more general deformation quantization of manifolds admitting an action of $\mathbb{R}^{2}$ due to Rieffel [50]. It relates also to the operation of twisting known it the theory of quantum groups.

However, it seems that it is yet quite premature to attempt any systematic treatment of quantum 4-spheres, and this is certainly beyond the scope of this note.

Acknowledgments. The author thanks the organizers for the invitation, the 'Geometric Analysis' Research Training Network HPRN-CT-1999-00118 of the European Commission for the financial support, P. M. Hajac and E. Hawkins for several comments on the manuscript, and M. A. Rieffel for the correspondence on the deformation quantization method.

\section{References}

[1] F. Bonechi, N. Ciccoli and M. Tarlini, Noncommutative instantons and the 4-sphere from quantum groups, Commun. Math. Phys. 226 (2002), 419-432.

[2] F. Bonechi, N. Ciccoli and M. Tarlini, Quantum even spheres $\Sigma_{q}^{2 n}$ from Poisson double suspension, preprint math.QA/0211462.

[3] O. Bratteli, G. A. Elliott, D. E. Evans and A. Kishimoto, Noncommutative spheres. I, Internat. J. Math. 2 (1991), 139-166.

[4] O. Bratteli, G. A. Elliott, D. E. Evans and A. Kishimoto, Noncommutative spheres. II. Rational rotations, J. Operator Theory. 27 (1992), 53-85.

[5] O. Bratteli and A. Kishimoto, Noncommutative spheres. III. Irrational rotations, Commun. Math. Phys. 147 (1992), 605-624.

[6] T. Brzeziński, Quantum homogeneous spaces as quantum quotient spaces, J. Math. Phys. 37 (1996), 2388-2399.

[7] T. Brzeziński, L. Dąbrowski and B. Zieliński, Hopf fibration and monopole connection over the contact quantum spheres, preprint math.QA/0301123.

[8] T. Brzeziński and C. Gonera, Non-commutative 4-spheres based on all Podleś 2-spheres and beyond, Lett. Math. Phys. 54 (2000/2001), 315-321.

[9] T. Brzeziński and S. Majid, Quantum group gauge theory on quantum spaces, Commun. Math. Phys. 157 (1993), 591-638. Erratum 167 (1995), 235. 
[10] R. J. Budzyński and W. Kondracki, Quantum principal fiber bundles: Topological aspects, Rep. Math. Phys. 37 (1996), 365-385, preprint 517 PAN, Warsaw, 1993, hep-th/9401019.

[11] D. Calow and R. Matthes, Covering and gluing of algebras and differential algebras, J. Geom. Phys. 32 (2000), 364-396.

[12] D. Calow and R. Matthes, Connections on locally trivial quantum principal fibre bundles, J. Geom. Phys. 41 (2002), 114-165.

[13] R. Chakrabarti and J. Segar, Jordanian quantum spheres, Modern Phys. Lett. A 16 (2001), 1731-1740.

[14] A. Connes, Gravity coupled with matter and the foundation of noncommutative geometry, Commun. Math. Phys. 182 (1996), 155-176.

[15] A. Connes, Cyclic cohomology, quantum group symmetries and the local index formula for $S U_{q}(2)$, preprint math.QA/0209142.

[16] A. Connes, M. Dubois-Violette, Noncommutative finite-dimensional manifolds. I. Spherical manifolds and related examples, Commun. Math. Phys. 230 (2002), 539-579.

[17] A. Connes and G. Landi, Noncommutative manifolds, the instanton algebra and isospectral deformations, Commun. Math. Phys. 221 (2001), 141-159.

[18] L. Dąbrowski, H. Grosse and P. M. Hajac, Strong connections and Chern-Connes pairing in the Hopf-Galois theory, Commun. Math. Phys. 220 (2001), 301-331.

[19] L. Dąbrowski and G. Landi, Instanton algebras and quantum 4-spheres, Diff. Geom. Appl. 16 (2002), 277-284.

[20] L. Dąbrowski, G. Landi and T. Masuda, Instantons on the quantum 4-spheres $S_{q}^{4}$, Commun. Math. Phys. 221 (2001), 161-168.

[21] D. A. Ellwood, A new characterisation of principal actions, J. Funct. Anal. 173 (2000), 49-60.

[22] L. D. Faddeev, N. Y. Reshetikhin and L. A. Takhtajan, Quantization of Lie groups and Lie algebras, Alg. Anal. 1 (1990), 178. [Leningrad Math. J. 1 (1990), 193]

[23] H. Grosse, J. Madore and H. Steinacker, Field theory on the q-deformed fuzzy sphere I, J. Geom. Phys. 38 (2001), 308-342.

[24] D. Gurevich, R. Leclercq and P. Saponov, Equivariant noncommutative index on braided sphere, preprint math.QA/0207268.

[25] P. M. Hajac, R. Matthes and W. Szymański, Quantum real projective space, disc and sphere, Algebras and Representation Theory.

[26] P. M. Hajac, R. Matthes and W. Szymański, A locally trivial quantum Hopf fibration, Algebras and Representation Theory, in press; preprint math.QA/0112317.

[27] P. M. Hajac, R. Matthes and W. Szymański, Graph $C^{*}$-algebras and $\mathbb{Z}_{2}$-quotients of quantum spheres, preprint math.QA/0209268.

[28] P. M. Hajac, R. Matthes and W. Szymański, Fredholm index and noncommutative Hopf fibrations, see http://info.fuw.edu.pl/ ${ }^{\sim} \mathrm{pmh} /$ access.html for the preliminary version.

[29] E. Hawkins, Quantization of equivariant vector bundles, Commun. Math. Phys. 202 (1999), $517-546$.

[30] J. H. Hong and W. Szymański, Quantum spheres and projective spaces as graph algebras, Commun. Math. Phys. 232 (2002), 157-188.

[31] S. Klimek and A. Leśniewski, A two-parameter quantum deformation of the unit disc, J. Funct. Anal. 115 (1993), 1-23.

[32] A. Kumjian, On the K-theory of the symmetrized non-commutative torus, C. R. Math. Rep. Acad. Sci. Canada 12 (1990), 87-89.

[33] J. Madore, The fuzzy sphere, Class. Quant. Grav. 9 (1992), 69. 
[34] I. L. Markov, $C^{*}$-algebra generated by a noncommutative circle, in: Appl. Meth. Funct. Anal. in Math. Phys., Berezhanskii (ed.), Kiev, 1991, 70-78 (in Russian).

[35] T. Masuda, Y. Nakagami and J. Watanabe, Noncommutative differential geometry on the quantum SU(2), I: An algebraic viewpoint, K-Theory 4 (1990), 157-180.

[36] T. Masuda, Y. Nakagami and J. Watanabe, Noncommutative differential geometry on the quantum two sphere of Podleś. I: An algebraic viewpoint, K-Theory 5 (1991), 151-175.

[37] K. Matsumoto, Non-commutative three dimensional spheres, Japan J. Math. 17 (1991), 333-356.

[38] K. Matsumoto, Non-commutative three dimensional spheres II-non-commutative Hopf fibering, Yokohama Math. J. 38 (1991), 103-111.

[39] K. Matsumoto and J. Tomiyama, Non-commutative lens spaces, J. Math. Soc. Japan 44 (1992), 13-41.

[40] G. Nagy, On the K-theory of the non-commutative circle, J. Oper. Theory 31 (1994), 303-309.

[41] G. Nagy and A. Nica, On the "quantum disc" and a "non-commutative circle", in: Algebraic Methods in Operator Theory, R. E. Curto and P. E. T. Jorgensen (eds.), Birkhäuser, Basel, 1994, 276-290.

[42] T. Natsume, On a continuous deformation of the 2-sphere, Talk at the MSRI, Apr 25 2001.

[43] T. Natsume and C. L. Olsen, Toeplitz operators on noncommutative spheres and an index theorem, Indiana Univ. Math. J. 46 (1997), 1055-1112.

[44] T. Natsume and C. L. Olsen, A new family of noncommutative 2-spheres, preprint (2002).

[45] R. Nest, Cyclic cohomology of a noncommutative sphere, Kopenhagen Univ. preprint (1989).

[46] H. Omori, Y. Maeda, N. Miyazaki and A. Yoshioka, Noncommutative 3-sphere: A model of noncommutative contact algebras, J. Math. Soc. Japan 50 (1998), 915-943.

[47] H. Omori, N. Miyazaki, A. Yoshioka and Y. Maeda, Noncommutative 3-sphere as an example of noncommutative contact algebras, in: Banach Center Publications 40, 1997, 329-334.

[48] P. Podleś, Quantum spheres, Lett. Math. Phys. 14 (1987), 521-531.

[49] M. A. Rieffel, $C^{*}$-algebras associated to irrational rotations, Pacific J. Math. 93 (1981), 415-429.

[50] M. A. Rieffel, Deformation quantization for actions of $R^{d}$, Mem. Amer. Math. Soc. 106 (1993), no. 506.

[51] M. A. Rieffel, Matrix algebras converge to the sphere for quantum Gromov-Hausdorff distance, Memoirs Amer. Math. Soc., in press; preprint math.OA/0108005.

[52] A. J-L. Sheu, Quantization of the Poisson SU(2) and its Poisson homogeneous space the 2-sphere, Commun. Math. Phys. 135 (1991), 217-232.

[53] A. Sitarz, More noncommutative 4-spheres, Lett. Math. Phys. 55 (2001), 127-131.

[54] A. Sitarz, Rieffel's deformation quantization and isospectral deformations, Int. J. Theor. Phys. 40 (2001), 1693.

[55] L. L. Vaksman and Y. S. Soibelman, Algebra of functions on quantum $S U(n+1)$ group and odd dimensional quantum spheres, Alg. Anal. 2 (1990), 101.

[56] J. C. Varilly, Quantum symmetry groups of noncommutative spheres Commun. Math. Phys. 221 (2001), 511-523.

[57] S. G. Walters, Projective modules over the Non-commutative Sphere, J. Lond. Math. Soc. 51 (1995), 589-602. 
[58] S. L. Woronowicz, Twisted SU(2) group. An example of a noncommutative differential calculus, Publ. RIMS, Kyoto University, 23 (1987), 117-181.

[59] S. Zakrzewski, Matrix pseudogroups associated with anti-commutative plane, Lett. Math. Phys. 21 (1991), 309-321.

[60] B. Zieliński, An idempotent for a Jordanian complex quantum sphere, this volume. 